\title{
Upregulated expression of ADAM12 is associated with progression of oral squamous cell carcinoma
}

\author{
ERIKA UEHARA $^{1^{*}}$, MASASHI SHIIBA $^{2^{*}}$, KEIJI SHINOZUKA $^{3}$, KENGO SAITO $^{4}$, YUKINAO KOUZU ${ }^{3}$, \\ HIROFUMI KOIKE ${ }^{3}$, ATSUSHI KASAMATSU ${ }^{2}$, YOSUKE SAKAMOTO ${ }^{2}$, KATSUNORI OGAWARA ${ }^{2}$, \\ KATSUHIRO UZAWA ${ }^{3}$ and HIDEKI TANZAWA ${ }^{2,3}$ \\ ${ }^{1}$ School of Medicine, Chiba University; ${ }^{2}$ Division of Dentistry and Oral-Maxillofacial Surgery, \\ Chiba University Hospital; Departments of ${ }^{3}$ Clinical Molecular Biology and \\ ${ }^{4}$ Molecular Virology, Graduate School of Medicine, Chiba University, \\ 1-8-1 Inohana, Chuo-ku, Chiba 260-8670, Japan
}

Received August 17, 2011; Accepted October 14, 2011

DOI: $10.3892 /$ ijo.2012.1339

\begin{abstract}
ADAMs are a disintegrin and metalloproteinase family of membrane-associated metalloproteinases characterized by their multidomain structure, and have been reported to be associated with various malignant tumors. The aim of this study was to identify crucial members of the ADAM family in oral squamous cell carcinoma (OSCC), and to reveal their biological function and clinical significance. To clarify whether ADAM family genes are involved in OSCC, changes in the expression profile were investigated by real-time quantitative reverse transcriptase-polymerase chain reaction (qRT-PCR) analysis and immunohistochemical analysis. Functional analysis was performed by comparing cellular proliferation of siADAM-transfected cell lines and parental cell lines. Real-time qRT-PCR analysis identified significantly upregulated expression of $A D A M 12$ in OSCC-derived cell lines. This was validated in OSCC samples using real-time qRT-PCR and immunohistochemical staining. ADAM12 expression was correlated with TNM classification; significantly greater expression of ADAM12 was observed in tumors with higher T classification and more advanced stages. Moreover, siADAM12-transfected cells showed both a suppressed proliferation rate and increased transforming growth factor (TGF)- $\beta 3$ expression. Our data indicate that ADAM12 is overexpressed in OSCC and might accelerate cellular proliferation. Its function may be associated with TGF- $\beta$ signaling. This study suggests that controlling the expression or activity of ADAM12 could be a useful strategy in the development of an effective cure for OSCC.
\end{abstract}

Correspondence to: Dr Masashi Shiiba, Division of Dentistry and Oral-Maxillofacial Surgery, Chiba University Hospital, 1-8-1 Inohana, Chuo-ku, Chiba 260-8677, Japan

E-mail: m.shiiba@faculty.chiba-u.jp

*Contributed equally

Key words: oral squamous cell carcinoma, (a) disintegrin and metalloproteinases, transforming growth factor $\beta 3$

\section{Introduction}

(A) disintegrin and metalloproteinases (ADAMs) are a family of membrane-associated metalloproteinases characterized by their multidomain structure (1). They have unique extracellular domains, including a prodomain, metalloproteinases domain, disintegrin domain, cysteine-rich domain, and epidermal growth factor (EGF)-like repeat domain, and they also possess a transmembrane domain and cytoplasmic domain. Through these domains, ADAMs exhibit many crucial biological activities. A disintegrin domain mediates cell-cell and cell-matrix interaction by binding integrins, and a cystein-rich domain is involved in binding some extracellular matrix (ECM) proteins. About half of ADAMs possess proteolytic activities thanks to their metallo-protease domain; these include sheddase activity, which can induce the cleavage of ectodomains of transmembrane proteins adjacent to the cell membrane (2). By shedding the ectodomain, many transmembrane proforms of growth factors, cytokines and chemokines, such as epidermal growth factor receptor (EGFR) ligand, tumor necrosis factor (TNF) $\alpha$, transforming growth factor (TGF) $\alpha$, and heparin-binding EGF-like growth factor (HB-EGF) are solubilized. This allows them to be used in paracrine and autocrine signaling pathways $(3,4)$. Furthermore, the majority of ADAMs have Src-homology 3 (SH3)-binding domain in their cytoplasmic tails, suggesting that ADAMs potentially activate signaling molecules with SH3 domain, such as Src and Grb2 $(5,6)$. Thus, it seems reasonable to think that ADAMs are closely related to tumorigenesis. Indeed, some have been reported to be up-regulated in various malignant tumors, including those of the lung, kidney, brain, breast, pancreas, skin, and liver, with a close relationship to parameters of tumor progression or prognosis $(1,5,7)$.

In the head and neck region, up-regulated mRNA or protein expressions of ADAM8, 9, 10, 12, 17, 28 have been found in oral squamous cell carcinoma (OSCC) samples (8-10). Takamune et al suggested that ADAM17 is involved in CD44 cleavage and is significantly correlated to nodal metastasis, local recurrence, and invasion in OSCC (11). Recently, 
Ge et al reported that both the sheddase activity and protein expression of ADAM17 were significantly higher in head and neck cancer tissues and cell lines than normal keratinocytes or oral mucosa, and that the sheddase activity was significantly associated with tumor size and recurrence rate, suggesting that activation of sheddase function is crucial in head and neck cancer (3). Moreover, Xu et al reported that ADAM9 is highly expressed in adenoid cystic carcinoma cell lines that possess a high metastatic potential (12). We previously reported upregulation of ADAM12 using DNA microarray analysis (13). These findings indicate that members of the ADAM family could be potentially target molecules for novel cancer therapies. However, little research has been performed concerning ADAMs in OSCC, and it remains unknown whether molecular targeted therapy using ADAMs is feasible in this tumor.

The aim of this study was to identify members of the ADAM family that are important in OSCC, and to reveal their biological function and clinical significance in terms of development of effective therapies.

\section{Materials and methods}

Cell lines and culture conditions. The OSCC-derived cell lines used in this study were HSC-2, HSC-3, HSC-4, SCC-4 (Human Science Research Resources Bank, Osaka, Japan), H1, and Sa3 (provided by Dr Fujita at Wakayama Medical University, Wakayama, Japan). All OSCC-derived cell lines were grown in Dulbecco's modified Eagle's medium/F-12 HAM (SigmaAldrich Co., St. Louis, MO, USA) supplemented with $10 \%$ fetal bovine serum (Sigma) and $50 \mathrm{U} / \mathrm{ml}$ of penicillin and streptomycin (Sigma). The institutional review board of Chiba University approved all relevant protocols. Five independent human normal oral keratinocyte (HNOK) cell lines were cultured and maintained in defined keratinocyte-SFM (Gibco Brl., Gaithusberg, Germany) (14). All cell lines were incubated at $37^{\circ} \mathrm{C}$ in a humidified atmosphere with $5 \% \mathrm{CO}_{2}$.

Tissue specimens. From patients who underwent surgery for primary OSCC at the Department of Dentistry and Oral Surgery, Chiba University Hospital between 1998 and 2009, we obtained 65 tissue samples. Cancer tissues and patient-matched normal epithelium were collected during tumor resection after the informed consent was obtained under a protocol reviewed and approved by the institutional review board of Chiba University. Postoperative follow-up data were collected until April 2011 or until the day of the patient's death, metastasis, or local recurrence.

The resected tissue samples were divided into two parts, one was frozen immediately after removal of the surrounding normal tissues and stored at $-80^{\circ} \mathrm{C}$ until RNA extraction; the other part was fixed in $10 \%$ buffered formaldehyde solution for histopathologic diagnosis and immunohistochemical (IHC) staining. Histopathologic diagnosis was performed by the Department of Pathology, Chiba University Hospital, using the World Health Organization criteria. Clinicopathologic staging followed the TNM classification of the International Union against Cancer. Histopathologically, all patients had squamous cell carcinoma, and tumor samples were checked to ensure that tumor tissue was present in more than $80 \%$ of the specimen.
Preparation of $c D N A$. Total RNA was extracted using TRIzol Reagent (Invitrogen Life Technologies, Carlsbad, CA, USA), according to the manufacturer's instructions. The quality of the total RNA was determined using a Bioanalyzer (Agilent Technologies, Palo Alto, CA, USA). Five micrograms of total RNA from each sample were reversed transcribed to cDNA using Ready-To-Go You-Prime First-Strand Beads (GE Healthcare, Little Chalfort, Buckinghamshire, UK) and oligo(dT) primer (Sigma Genosys, Hokkaido, Japan), according to the manufacturers' protocols.

mRNA expression analysis using real-time quantitative reverse transcriptase-polymerase chain reaction ( $q R T-P C R)$. Real-time qRT-PCR was performed to validate mRNA expression with a single method using a LightCycler FastStart DNA Master SYBR Green 1 Kit (Roche Diagnostics GmbH, Mannheim, Germany), according to the procedure provided by the manufacturer. The nucleotide sequences of gene-specific primers for real-time qRT-PCR analysis are shown in Table I. PCR reactions using this apparatus were carried out in a final volume of $20 \mu \mathrm{l}$ of a reaction mixture consisting of $2 \mu \mathrm{l}$ of FirstStart DNA Master SYBR Green I mix (Roche), $3 \mathrm{mM} \mathrm{MgCl}_{2}$, and $0.2 \mu \mathrm{l}$ of the primers, according to the manufacturer's instructions. We then loaded the reaction mixture into glass capillary tubes for initial denaturation at $95^{\circ} \mathrm{C}$ for $10 \mathrm{~min}$, followed by 33-45 rounds of amplification at $95^{\circ} \mathrm{C}(10 \mathrm{sec})$ for denaturation, $62-68^{\circ} \mathrm{C}(10 \mathrm{sec})$ for annealing, and $72^{\circ} \mathrm{C}$ for extension, with a temperature slope of $20^{\circ} \mathrm{C} / \mathrm{sec}$. This was performed in the LightCycler. The transcript amount was estimated from the respective standard curves and normalized to that of glyceraldehyde-3-phosphate dehydrogenase (GAPDH). The sequence of the specific primer for GAPDH was 5'-CATCTCTGCCCCCTCTGCTGA-3' (forward) and 5'-GGATGACCTTGCCCACAGCCT-3' (reverse).

Immunohistochemical analysis. Immunohistochemical staining was performed on $4-\mu \mathrm{m}$ sectionsof paraffin-embedded specimens using rabbit anti-ADAM12 polyclonal antibody (Vector Laboratories, Inc., Burlingame, CA, USA) (ADAM12 was found to have increased expression in OSCC-derived cell lines). Briefly, after deparaffinization and hydration, the slides were treated with endogenous peroxidase in $0.3 \%$ hydrogen peroxide for $30 \mathrm{~min}$, after which they were blocked for $2 \mathrm{~h}$ at room temperature with $1.5 \%$ blocking serum (Santa Cruz Biotechnology, Santa Cruz, CA, USA) in PBS before reacting with anti-ADAM12 antibody (1:100 dilution) at room temperature in a moist chamber overnight. Upon incubation with the primary antibody, the specimens were washed and treated with EnVision reagent (Dako Japan, Tokyo, Japan) followed by color development in 3,3'-diaminobenzidine tetrahydrochloride (Dako). Finally, the slides were lightly counterstained with hematoxylin, dehydrated with ethanol, cleaned with xylene, and mounted. To obtain a quantitative measure of the ADAM12 protein expression level, a scoring method was used in which the mean percentage of positive tumor cells was determined in at least five random fields at magnification $\mathrm{x} 400$ in each section. The intensity of the ADAM12 immunoreaction was scored as follows: $1+$, weak; $2+$, moderate; and $3+$, intense. The percentage of positive tumor cells and the staining intensity then were multiplied to produce the immunohistochemical staining score (IHC score) $(15,16)$. The maximum immunohistochemical 
Table I. Specific primer sequences used in real-time qRT-PCR analysis.

\begin{tabular}{ll}
\hline Target gene & \multicolumn{2}{c}{ Primer sequence } \\
\hline ADAM9 & (F) 5'-GGACCAATGGAAACCTGATCAA-3' \\
ADAM10 & (R) 5'-ACTGTCATGTCTCCGACGTGTG-3' 5 '-CGTTTCCCAAATATTGGTGTGG-3' \\
& (R) 5'-CAGACCAAGTACGCCATCATCA-3' \\
ADAM12 & (F) 5'-AAATCCCATGACAATGCGCAG-3' \\
& (R) 5'-CATGACAATTCCCCCAGACTGG-3' \\
ADAM17 & (F) 5'-TCCAGTGCAGTGACAGGAACA-3' \\
ADAM28 & (F) 5'-CACGCCTTTGCAAGTAGCATTA-3' \\
& (R) 5'-AGATCACCACAAGCCCACAAA-3' \\
ADAM33 & (F) 5'-CCCAGAAGCAAAGGTCACACA-3' \\
TGF 33 & (R) 5'-CCGTGGAAATTGCATGTAGGA-3' \\
& (F) 5'-CTGGCCCTTTACAACAGC-3' \\
& (R) 5'-CACTGAGGACACATTGAAGC-3'
\end{tabular}

staining score was 156.9 in normal tissues, therefore, cases in which the score was $>156.9$ were considered positive. Scoring was done by two investigators, neither of whom had knowledge of the patients' clinical status.

Protein extraction. Protein was extracted from the cells when they reached $80-90 \%$ confluence. To do this, the cells were washed twice with phosphate-buffered saline (PBS), scraped into a tube, and centrifuged briefly. We then incubated the cell pellets at $4^{\circ} \mathrm{C}$ for $30 \mathrm{~min}$ in a lysis buffer (7 M urea, $2 \mathrm{M}$ thiourea, $4 \%$ w/v CHAPS, and $10 \mathrm{mM}$ Tris $\mathrm{pH}$ 8.0) with proteinase inhibitor cocktail (Roche). Protein concentration was determined using a commercial Bradford reagent (Bio-Rad, Richmond, CA, USA) and adjusted to $1 \mathrm{mg} / \mathrm{ml}$ with lysis buffer.

Western blot analysis. Samples $(20 \mu \mathrm{g})$ of proteins were separated by SDS/PAGE in 4-12\% gel and transferred to nitrocellulose membranes for reaction with $1 \mu \mathrm{g} / \mathrm{ml}$ affinity-purified rabbit anti-ADAM12 polyclonal antibody (Vector Laboratories), and $1 \mu \mathrm{g} / \mathrm{ml}$ affinity-purified mouse anti-human $\beta$-actin monoclonal antibody (Abcam, Cambridge, MA, USA). Secondary antibodies, horseradish peroxidase (HRP)-conjugated anti-rabbit or antimouse immunoglobulin (Ig)G, were detected using SuperSignal Chemiluminescent substrate (Pierce Biotechnology, Rockford, IL, USA).

Transfection with small-interfering RNA (siRNA). SMARTpool siRNA targeting ADAM12 (siADAM12; SMARTpool, M-016684), and positive and negative control siRNAs were used in the experiment (Dharmacon, Lafayette, CO, USA). Two negative controls were used, a vehicle control and a siControl non-targeting iRNA pool (D-001206-13-20; siNT). Cyclophilin B (siControl cyclophilin B; siCyclo) was used as a positive silencing control to ascertain the transfection efficiency in each experiment. Cells were transfected with siRNAs using DharmaFECT1 siRNA transfection reagent (Dharmacon).

Cellular proliferation assay. To determine the effect of ADAM12 siRNA on cell proliferation, HSC-3 cells transfected with non-targeting or ADAM12 siRNA (100 nmol/1) were seeded in 12-well plates at a density of $1 \times 10^{4}$ viable cells per well. Mock-transfected cells were treated with DharmaFECT1 reagent but not siRNA, as vehicle controls. At the indicated time point, cells were trypsinized and counted using a hemocytometer in triplicate samples. The effect of ADAM12 gene silencing on cell proliferation was investigated in the same manner for $\mathrm{H} 1$ cells.

Analysis of transforming growth factor (TGF)- $\beta 3 \mathrm{mRNA}$ expression in ADAM12 knock-down cells. TGF- $\beta$ is a wellknown multifunctional molecule mediating various cellular process, including cellular proliferation. Verona et al reported that $A D A M 12$ is one of the genes up-regulated in response to TGF- $\beta 3$ treatment in prostate stromal cells, suggesting that TGF- $\beta$ signaling may regulate ADAM12 expression (17). Thus, to evaluate the relationship between ADAM12 and TGF- $\beta 3$, expression of $T G F-\beta 3 \mathrm{mRNA}$ in $A D A M 12$ knock-down cells was examined.cDNA was synthesized from total RNA extracted from siADAM12- or siNT-transfected OSCC-derived cell lines (HSC-3 and H1), and was subjected to real-time qRT-PCR analysis. The nucleotide sequences of specific primers for TGF- $\beta 3$ are shown in Table I.

Statistical analysis. In comparisons of ADAM12 expression levels, statistical significance was evaluated by Mann-Whitney's U test. Relationships between ADAM12-immunohistochemical staining scores and clinicopathological profiles were evaluated by $\chi^{2}$ test, Fisher's exact test, and Mann-Whitney's U test. The criterion for statistical significance was $\mathrm{P}<0.05$.

\section{Results}

$m R N A$ expression analysis. We examined the mRNA expression levels of the six ADAM genes (ADAM9, ADAM10, ADAM12, $A D A M 17, A D A M 28$ and $A D A M 33)$ that showed significance in DNA microarray analysis performed previously $(13,16)$. The present study revealed higher expression of $A D A M 12$ mRNA in all OSCC cell lines examined when compared with HNOKs (Fig. 1). A tendency for decreased expression of ADAM33 mRNA in OSCC cell lines was also shown. These results were consistent with the data found in our previous DNA microarray analysis $(13,16)$. Since mRNA expressions of other members of the ADAM family varied in OSCC cell lines examined, definitive conclusions could not be obtained. Our data strongly suggested an important role for ADAM12 in OSCC, so this molecule was investigated further.

To confirm the expression profile of ADAM12, mRNA expression level was determined by real-time qRT-PCR analysis in 65 OSCC samples obtained from patients with primary OSCC. When compared with matched normal tissues, mRNA expression level of $A D A M 12$ was significantly increased. The median relative expression level of $A D A M 12$ was significantly higher in OSCC compared with normal tissues (1.27 vs. 0.11, $\mathrm{P}<0.001$ ) (Fig. 2). 

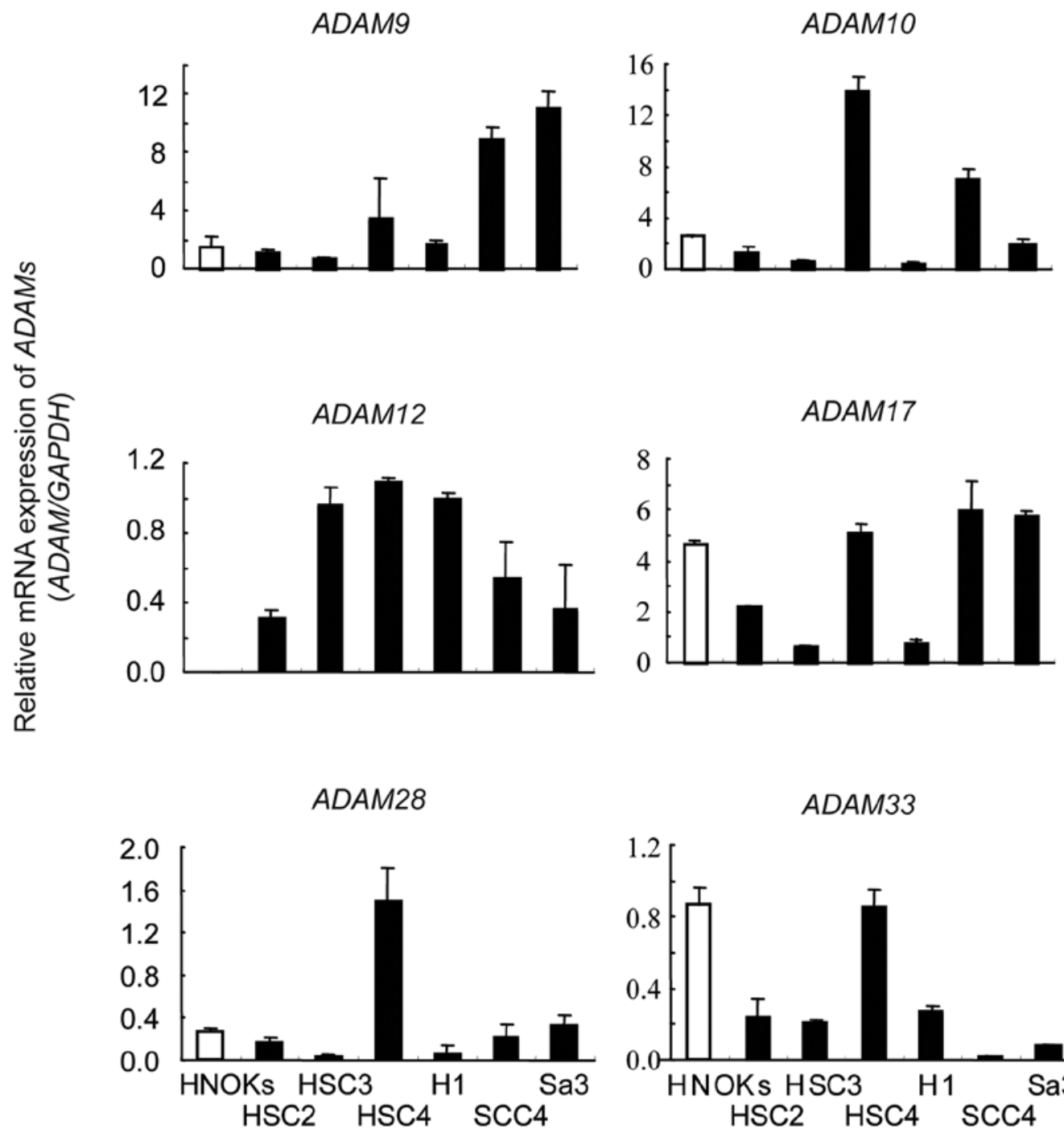

Figure 1. the mRNA expression status of ADAM genes in OSCC-derived cell lines. Quantification of mRNA levels in OSCC-derived cell lines by real-time qRT-PCR analysis. Significant up-regulation of ADAM12 mRNA expression was found in all OSCC-derived cell lines examined. Data are expressed as the mean \pm standard deviation $(\mathrm{SD})$.

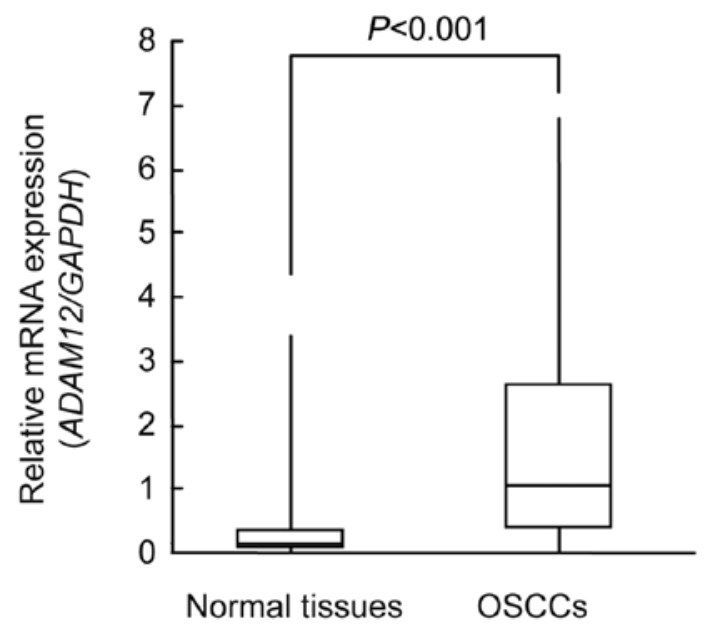

Figure 2. ADAM12 mRNA expression status in primary OSCC samples. Relative mRNA expressions of ADAM12 in 65 OSCC samples and matched normal tissues were determined using real-time qRT-PCR and compared. The expression level was analyzed by Mann-Whitney's U test, and the median relative expression level of ADAM12 mRNA was significantly higher in OSCC compared with normal tissues (1.27 vs. $0.11, \mathrm{P}<0.001)$.
Immunohistochemical analysis. On immunohistochemical analysis using anti-ADAM12 antibody, distinct positive signals were detected in OSCC samples, on the other hand, only weak staining was observed in normal tissues (Fig. 3). Median IHC score was significantly lower in normal tissues than in OSCC samples (48.0 vs. 214.1, $\mathrm{P}<0.001$ ) (Fig. 4A). Furthermore, statistically significant differences of ADAM12 expression were found for tumor stage: between the T1 or T2 group and T3 or T4 group (Fig. 4B), and between early stage group (stage I or II) and advanced stage group (stage III or IV) (Fig. 4C). In the present study, cases with an IHC score higher than the maximum score (156.9) in the normal tissue group were considered positive for ADAM12. According to this criterion, 52 cases showed significantly up-regulated ADAM12 expression. To assess whether ADAM12 could be a useful prognostic biomarker, the relationship between clinical factors and ADAM12 expression was evaluated. ADAM12 expression was positive in $67 \%$ of T1 or T2 tumors, $87 \%$ of T3 tumors, and $93 \%$ of T4 tumors. There was a statistically significant relationship between ADAM12 expression and primary tumor size $(\mathrm{P}=0.0191)$ (Table II), 
A

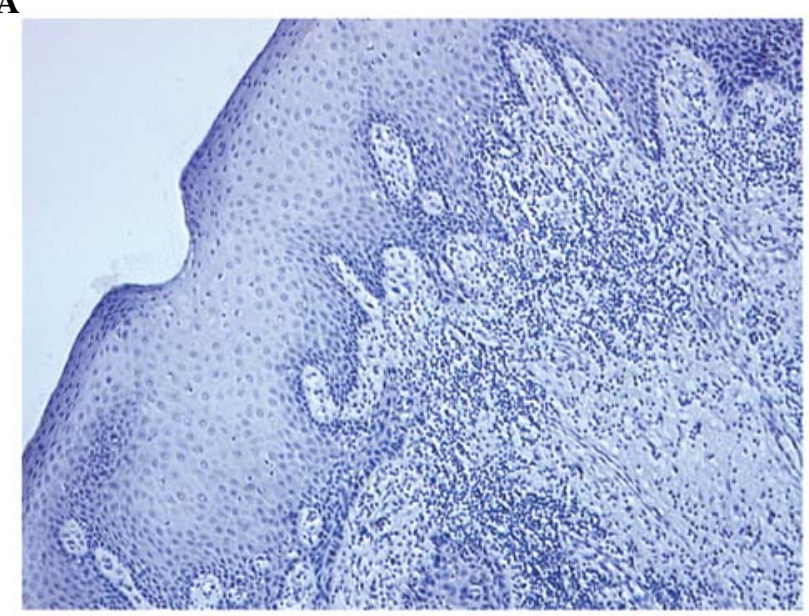

B

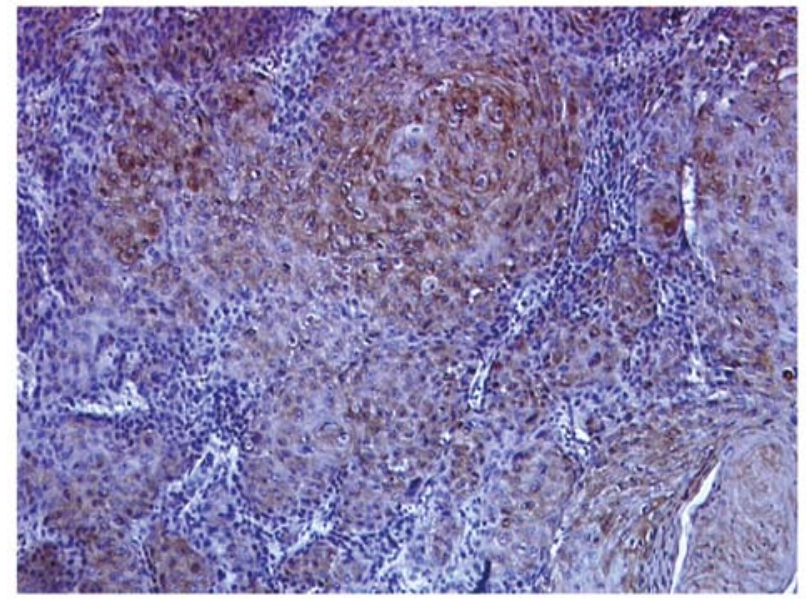

Figure 3. Immunohistochemical staining of ADAM12 in primary OSCC and normal tissue. Specimens from OSCCs and normal tissues were stained with anti-ADAM12 antibody. Representative results of ADAM12 protein expression in normal tissue (A) and primary OSCC tissue (B) are shown. Strong immunoreactivity was detected in OSCC tissue, whereas only weak staining was observed in normal tissues.

with higher ADAM expression in larger tumors (T3 or T4) when compared with smaller tumors (T1 or T2) $(\mathrm{P}=0.0268)$ (Table II). Furthermore, ADAM12 expression was significantly higher in the advanced stage group than in the early stage group $(\mathrm{P}=0.0028)$, suggesting that ADAM12 might be closely associated with tumor development.

Cell proliferation assay. To examine the role of ADAM12 in cellular proliferation, non-targeting (siNT) or ADAM12 siRNA was introduced into HSC-3 and H1 cells. Expression levels of ADAM12 protein were confirmed in the controls and transfectants through Western blot analysis. We found obvious ADAM12 protein expression in the control (non-treatment parental cell), vehicle, and siNT cells, but expression was clearly diminished in siADAM12-transfected cells (Fig. 5). When siADAM12transfected cells were subjected to the proliferation assay, their proliferation rate was significantly lower than that of the control, vehicle and siNT group (Fig. 6).

Relationship between ADAM expression andTGF- $\beta 3$ expression. When expression of TGF- $\beta 3$ mRNA in ADAM12 knock-down
A

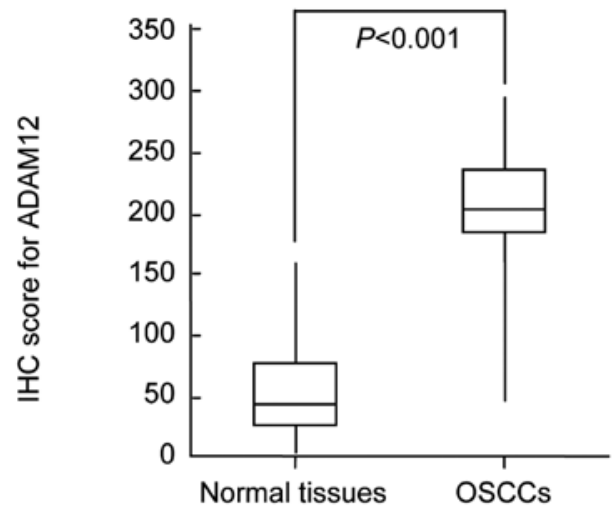

B

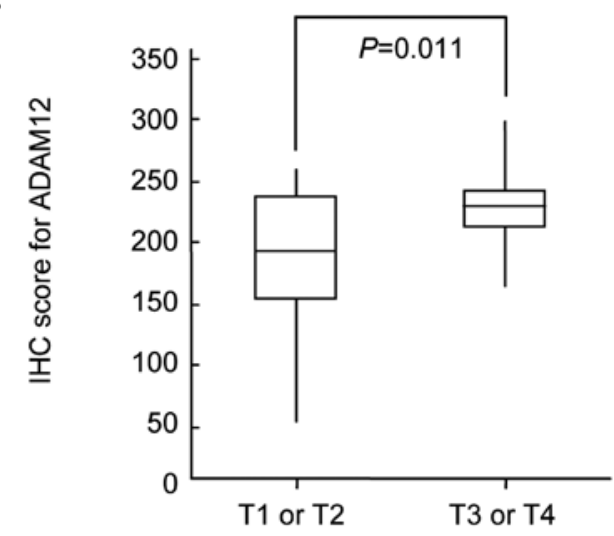

C

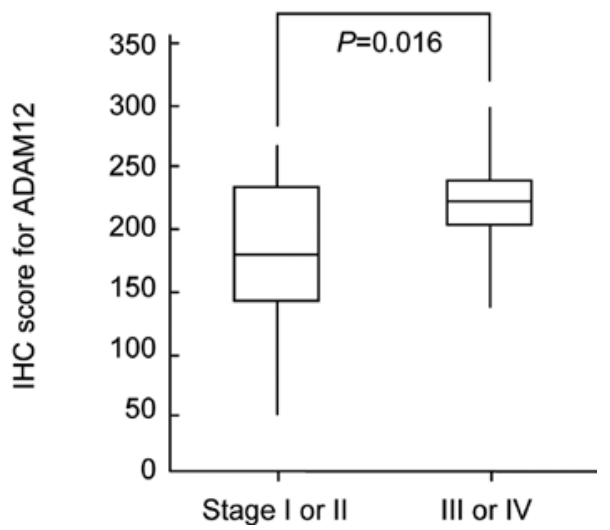

Figure 4. Analysis of immunohistochemical staining data in OSCC samples and normal tissues. Expression level of ADAM12 determined by immunohistochemical staining was calculated as an IHC score and analyzed by Mann-Whitney's U test. (A) IHC score for ADAM12 obtained from 65 OSCC samples was significantly higher than that in matched normal tissues $(\mathrm{P}<0.001)$. (B) Association between ADAM12 protein expression and tumor size. IHC score for ADAM12 was significantly higher for T3 or T4 cases than for $\mathrm{T} 1$ or $\mathrm{T} 2$ cases $(\mathrm{P}=0.011)$. (C) Comparison of ADAM12 protein expression in patients with early-stage disease (stages I or II) OSCC and patients with advanced-stage (stages III or IV) OSCC. ADAM12 protein expression in the advanced stage group is significantly higher than in the early stage group $(\mathrm{P}=0.016)$.

cells was examined using real-time qRT-PCR analysis, higher expressions of $T G F-\beta 3$ mRNA were observed in siADAM12transfected cells when compared with the parental cell lines (Fig. 7). TGF- $\beta 3$ mRNA expression in siADAM12-transfected cells was 3.3 times greater than that in parental cells for HSC-3 cells (Fig. 7A) and 2.4 times greater for $\mathrm{H1}$ cells (Fig. 7B). 
Table II. Correlation between ADAM12 expression and clinicopathological profiles.

\begin{tabular}{|c|c|c|c|c|}
\hline \multirow[b]{2}{*}{ Clinical factor } & \multirow[b]{2}{*}{ Total } & \multicolumn{2}{|c|}{ Number of cases $(\%)$} & \multirow[b]{2}{*}{ P-value ${ }^{a}$} \\
\hline & & ADAM12(-) & $\operatorname{ADAM} 12(+)$ & \\
\hline \multicolumn{5}{|l|}{ Age at surgery (years) } \\
\hline$<60$ & 14 & $3(21)$ & $11(79)$ & \multirow{3}{*}{$0.8747^{b}$} \\
\hline$\geq 60,<70$ & 17 & $4(24)$ & $13(76)$ & \\
\hline$\geq 70$ & 34 & $6(18)$ & $28(82)$ & \\
\hline \multicolumn{5}{|l|}{ Gender } \\
\hline Male & 43 & $10(23)$ & $33(77)$ & \multirow{2}{*}{$0.5162^{\mathrm{c}}$} \\
\hline Female & 22 & $3(14)$ & $19(86)$ & \\
\hline \multicolumn{5}{|l|}{ Primary tumor size } \\
\hline $\mathrm{T} 1$ & 12 & $4(33)$ & $8(67)$ & \multirow{4}{*}{$0.0191^{\mathrm{d}}$} \\
\hline $\mathrm{T} 2$ & 18 & $6(33)$ & $12(67)$ & \\
\hline $\mathrm{T} 3$ & 8 & $1(13)$ & $7(87)$ & \\
\hline $\mathrm{T} 4$ & 27 & $2(7)$ & $25(93)$ & \\
\hline $\mathrm{T} 1+\mathrm{T} 2$ & 30 & $10(33)$ & $20(67)$ & \multirow{2}{*}{$0.0268^{c}$} \\
\hline $\mathrm{T} 3+\mathrm{T} 4$ & 35 & $3(9)$ & $32(91)$ & \\
\hline \multicolumn{5}{|c|}{ Regional lymph node metastasis } \\
\hline $\mathrm{N}(-)$ & 37 & $8(22)$ & $29(78)$ & \multirow{2}{*}{$0.4787^{c}$} \\
\hline $\mathrm{N}(+)$ & 28 & $5(18)$ & $23(82)$ & \\
\hline \multicolumn{5}{|l|}{ Stage } \\
\hline $\mathrm{I}$ & 11 & $3(27)$ & $8(73)$ & \multirow{4}{*}{$0.0694^{\mathrm{d}}$} \\
\hline II & 9 & $5(56)$ & $4(44)$ & \\
\hline III & 7 & $0(0)$ & $7(100)$ & \\
\hline IV & 38 & $5(13)$ & $33(87)$ & \\
\hline $\mathrm{I}+\mathrm{II}$ & 20 & $8(40)$ & $12(60)$ & \multirow{2}{*}{$0.0028^{\mathrm{c}}$} \\
\hline $\mathrm{III}+\mathrm{IV}$ & 45 & $5(11)$ & $40(89)$ & \\
\hline \multicolumn{5}{|l|}{ Histopathologic type } \\
\hline Well differentiated & 44 & $7(16)$ & $37(84)$ & \multirow{3}{*}{$0.1977^{\mathrm{d}}$} \\
\hline Moderately differentiated & 16 & $4(25)$ & $12(75)$ & \\
\hline Poorly differentiated & 5 & $2(40)$ & $3(60)$ & \\
\hline
\end{tabular}

${ }^{a} \mathrm{P}<0.05$ was considered significant, ${ }^{\mathrm{b}} \chi^{2}$ test, ${ }^{\mathrm{c}}$ Fisher's exact test, ${ }^{\mathrm{d}}$ Mann-Whitney's U test.

\section{Discussion}

In the present study, up-regulated expression of $A D A M 12$ mRNA was detected in OSCC cell lines and primary OSCC tissue samples, and the increase of ADAM12 protein expression was confirmed by IHC of OSCC specimens. The up-regulated expression of ADAM12 protein was obvious in advanced tumors, and proliferation of OSCC-derived cell lines was suppressed when the ADAM12 gene was knocked down. These data suggest that ADAM12 expression is associated with cellular proliferation in OSCC.

ADAM12 is a member of the ADAM family, and possesses catalytically active sites in its metalloproteinase domain. Other extracellular domains of ADAM12 are a disintegrin domain, cysteine-rich domain, and EGF-like domain (7). There are two splice variants of human ADAM12: ADAM12-L and ADAM12-S. ADAM12-L has transmembrane domain and cytoplasmic domain, and exists as a membrane anchored form; in contrast, ADAM12-S lacks these domains and is secreted as a soluble form. Through these domains, ADAM12 exhibits crucial biological functions and is associated with various diseases including cancers. Overexpression of ADAM12 has been demonstrated in tumors of the breast $(18,19)$, bladder $(20)$, liver (21), lung (22), stomach (23), brain (24), and bone (25).

Kornberg et al reported up-regulated expression of ADAM12 in OSCC using real-time PCR and IHC analysis (9), however, the functional roles of ADAM12 in OSCC has not yet been clarified. In the present study, in addition to up-regulated expression 
A Control Vehicle siNT siADAM12

ADAM12

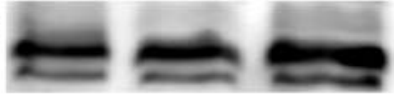

$\beta$-actin

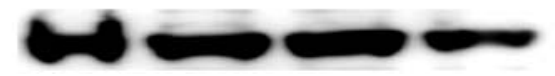

B

Control Vehicle siNT siADAM12

ADAM12

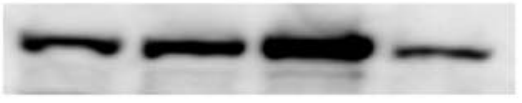

$\beta$-actin

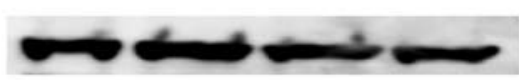

Figure 5. Detection of ADAM12 protein in siADAM12-transfected cells. Expressions of ADAM12 protein in siADAM12-transfected OSCC cells and control cells were analyzed with Western blotting. Significant diminished expression of ADAM12 protein was shown in siADAM12-transfected HSC-3 cells (A) and in siADAM12-transfected H1 cells (B).

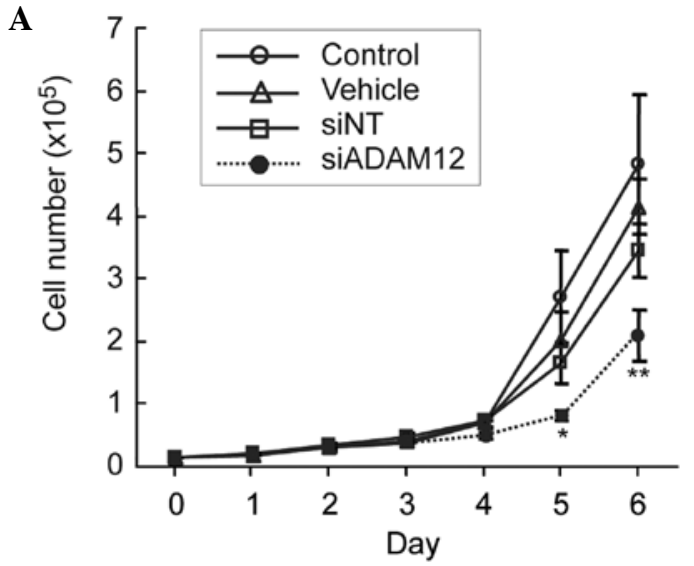

$\mathbf{B}$

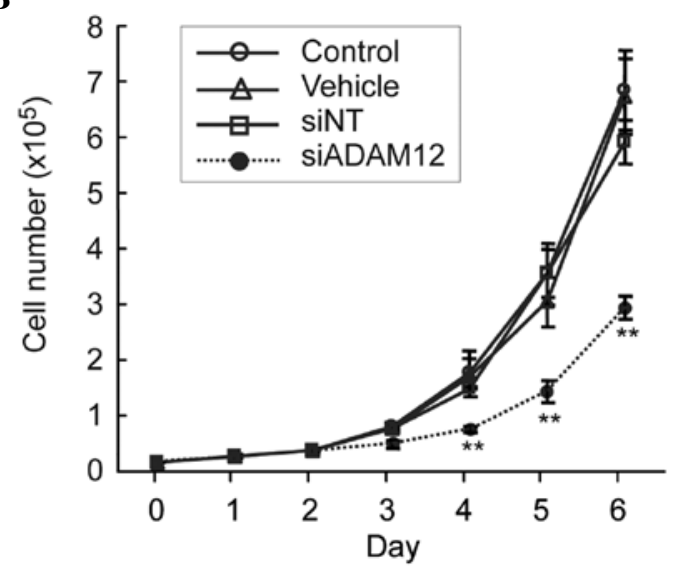

Figure 6. Cellular proliferation of siADAM12-transfected cells. A cellular proliferation assay was performed to investigate the effect of diminished ADAM12 expression on OSCC-derived cell lines, HSC-3 (A) and H1 (B). The proliferation rate of siADAM12-transfected cells was significantly suppressed when compared with control cells. The asterisks indicate significant differences between siNT and siADAM12 $\left({ }^{*} \mathrm{P}<0.05,{ }^{* *} \mathrm{P}<0.01\right.$, Student's t-test $)$.

of ADAM12 in OSCC, we found that OSCC-derived cell lines exhibited suppressed proliferation when the ADAM12 gene

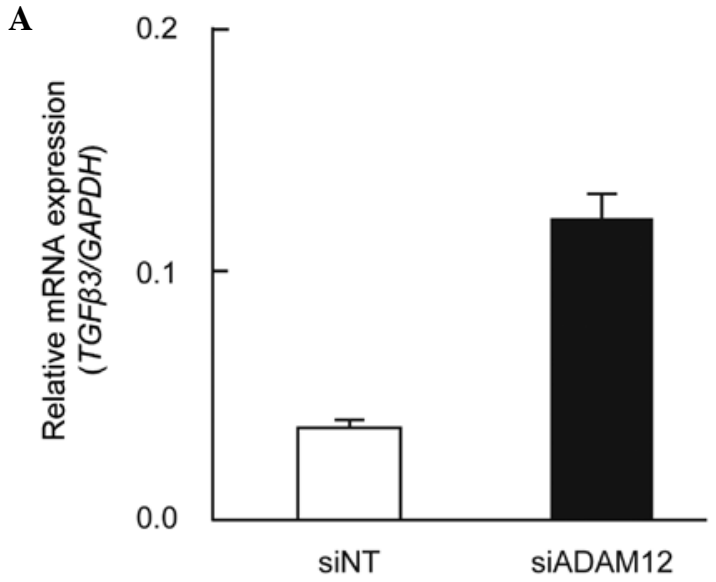

B

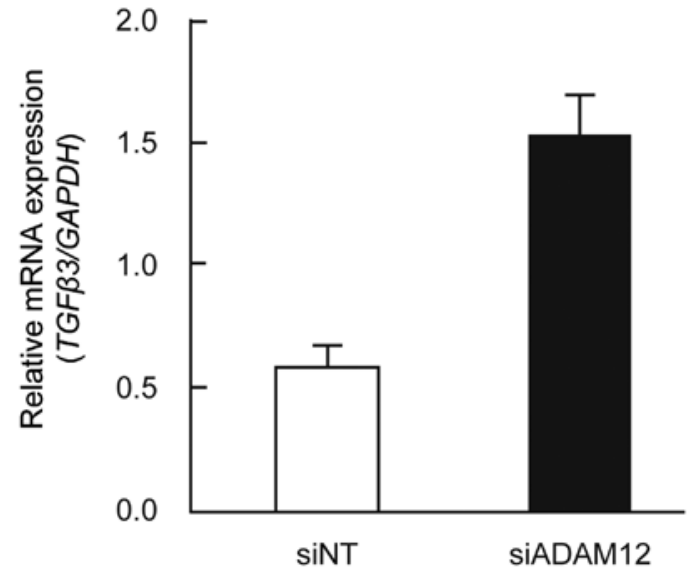

Figure 7. Effect of mRNA silencing of ADAM12 on TGF- $\beta 3$ expression in OSCC cell lines. After mRNA silencing of ADAM12 by siADAM12 transfection of OSCC-derived cell lines, analysis of $T G F-\beta 3$ mRNA expression in OSCC-derived cell lines was performed using real-time qRT-PCR and expression levels were compared between siADAM12-transfected and siNTtransfected cells. Significant up-regulated expressions of $T G F-\beta 3$ mRNA were found in both siADAM12-transfected HSC-3 (A) and siADAM12-transfected H1 (B), with levels increasing by 3.3 and 2.4 times, respectively.

was silenced by introducing siADAM12. Taken together with our finding of a significant relationship between up-regulated ADAM12 expression and primary tumor size, these data strongly suggest that ADAM12 positively regulates cellular proliferation in OSCC.

Detailed mechanisms of how ADAMs influence cancer biology is gradually elucidated. Mochizuki $e t$ al sug-gested five potential ways in which ADAMs could be involved in cancer biology: i) activation of proADAMs by furin, MMPs, or other unknown pathways, ii) activation of growth factors such as TGF- $\alpha$ and HB (heparin-binding)-EGF by the sheddase activities of ADAMs, resulting in enhanced cellular proliferation by paracrine and autocrine mechanisms, iii) binding to integrins or syndecans via the disintegrin and cysteine-rich domains of ADAMs, which may help cells to cleave substrates such as extracellular matrix, iv) promotion of cell proliferation signals through integrins, and v) processing undetermined membrane-anchored molecules such as chemokines, cytokines, and their receptors (5). Among these potential activities, the ectodomain shedding of 
HB-EGF has been well investigated. Kodama et al showed that in glioblastoma, $A D A M 12$ mRNA is selectively up-regulated in 13 different ADAMs. They found that a soluble form of HB-EGF was observed in association with this ADAM12 expression and that there was a direct correlation between the mRNA expression levels of ADAM12 and proliferative activity. They suggested that ADAM12 potentially plays a role in the prominent proliferation of glioblastomas through shedding of HB-EGF (24). Eve-1 and PACSIN3 have been identified as ADAM12 binding proteins and are involved in HB-EGF shedding $(26,27)$. This shedding mediates transactivation of EGFR and regulates EGFR activity. EGFR-associated signaling is one of the major proliferative pathways, and dysregulation of this signaling could induce tumorigenesis (28). Thus, it is reasonable to speculate that up-regulated expression of ADAM12 may induce HB-EGF shedding and enhance proliferative signals through EGFR in OSCCs.

Recently, Ebi et al demonstrated the mechanism of proHBEGF shedding and EGFR phosphorylation through ADAM17 in gastric cancer (29). They suggested that a complex of TGF- $\beta 1$ and its receptors (T $\beta$ RI and T $\beta$ RII) activates ADAM17, and that activated ADAM induces proHB-EGF shedding, resulting in EGFR transactivation. Atfi et al demonstrated that ADAM12 positively regulates the TGF- $\beta$ signaling pathway, including phosphorylation of Smad2, association of Smad2 with Smad4, and activation of transcription, by facilitating the trafficking of T $\beta R I I$ to endosomes (30). These studies indicate that activation of EGFR signaling induced by ADAM12-mediated shedding of EGFR ligands may play crucial roles in cancer development, and that there might be a close relationship between ADAM12 protease activity and TGF- $\beta$ signaling. Many studies have reported that TGF- $\beta$ has complex biological functions, and now it is believed that TGF- $\beta$ acts upon the epithelium as a tumor suppressor in the early tumorigenesis stage and TGF- $\beta$ signaling has been associated with late tumor progression $(31,32)$. In the present study, we found that the proliferation of siADAM12transfected OSCC-derived cell lines was suppressed when TGF- $\beta 3$ expression was elevated. Thus, TGF- $\beta$ signaling may negatively regulate cellular proliferation of OSCC-derived cell lines in our experimental condition. Although the data did not directly prove functions of TGF- $\beta$ signaling in OSCCs, ADAM12 and TGF- $\beta$ appear to influence each other.

TGF- $\beta$ has the ability to modulate E-cadherin expression and induce reversible epithelial to mesenchymal transdifferentiation (EMT) in epithelial cells (33). Qiao et al recently demonstrated that Snail and Slug act as regulators of EMT induced by TGF- $\beta 1$ in OSCC cell lines (34). However, although EMT in cancer has attracted attention, to the best of our knowledge there is no published research investigating associations between ADAMs and EMT induced by TGF- $\beta$. Thus, further research into this area should yield information important in the understanding of OSCC tumorigenesis.

Besides T $\beta$ RII, follistatin-related gene (FLRG) (35), syndecan-4 (36), and integrins (37) are known to bind extracellularly with the cysteine-rich or disintegrin domains of ADAM12. These interactions could help the degradation of ECM, leading to cancer invasion. The tendency toward advanced progression observed in ADAM12-positive OSCC cases in the present study might be due to the effects of ADAM12 on the ECM. Moreover, intercellular signaling molecules such as
Src (38), PACSIN3 (26), Eve-1 (27), PI3-K, p85a (39), and Grab2 (6) interact with proline-rich SH3-binding sites in the tail of ADAM12. Based on these facts, it is reasonable to think that ADAM12 potentially regulates various signaling pathways, not only proliferative signals, in OSCC.

Since ADAM12 causes ectodomain shedding of EGFR ligands, inhibiting its activity might be a novel clinical approach to modulating ErbB family signaling in cancer. Fridman et al reported that a synthesized and selective inhibitor of the sheddase activity of ADAMs can prevent the release of ErbB ligands, leading to inhibition of ErbB signaling pathways (40). Recent studies suggested that some tissue inhibitor of metalloproteinases (TIMPs) such as TIMP-3, TIMP-2, and TIMP-1 exhibit inhibitory activity against ADAM12, and that a highly selective inhibitor for ADAM12 catalytic activity could be designed by engineering TIMP-2 $(41,42)$. Although a selective ADAM12 inhibitor for use in OSCC has not yet been developed, judging from our results, it might be possible to use an ADAM12 inhibitor to suppress cellular proliferation of OSCCs, leading to improved clinical outcome.

In conclusion, the present study demonstrated that expression of ADAM12 is significantly increased in OSCC-derived cell lines and primary OSCC samples. IHC revealed that ADAM12 expression was associated with both $\mathrm{T}$ (primary tumor size) and stage classification. Furthermore, ADAM12 knockdown OSCC cell lines showed diminished proliferation accompanied by up-regulated $T G F-\beta 3$ expression. These results strongly suggest that ADAM12 positively regulates proliferation of OSCCs, probably through its protease and adhesion activities, and that TGF- $\beta$ signaling might be associated with ADAM12 functions.

\section{References}

1. Murphy G: The ADAMs: signalling scissors in the tumour microenvironment. Nat Rev Cancer 8: 929-941, 2008.

2. Klein T and Bischoff R: Active metalloproteases of the A disintegrin and metalloprotease (ADAM) family: biological function and structure. J Proteome Res 10: 17-33, 2010.

3. Ge L, Baskic D, Basse P, et al: Sheddase activity of tumor necrosis factor- $\alpha$ converting enzyme is increased and prognostically valuable in head and neck cancer. Cancer Epidemiol Biomarkers Prev 18: 2913-2922, 2009.

4. Horiuchi K, Le Gall S, Schulte M, et al: Substrate selectivity of epidermal growth factor-receptor ligand sheddases and their regulation by phorbol esters and calcium influx. Mol Biol Cell 18: 176-188, 2007.

5. Mochizuki S and Okada Y: ADAMs in cancer cell proliferation and progression. Cancer Sci 98: 621-628, 2007.

6. Suzuki A, Kadota N, Hara T, et al: Meltrin $\alpha$ cytoplasmic domain interacts with $\mathrm{SH} 3$ domains of Src and Grb2 and is phosphorylated by v-Src. Oncogene 19: 5842-5850, 2000.

7. Kveiborg M, Albrechtsen R, Couchman JR and Wewer UM: Cellular roles of ADAM12 in health and disease. Int J Biochem Cell Biol 40: 1685-1702, 2008.

8. Ko SY, Lin SC, Wong YK, Liu CJ, Chang KW and Liu TY: Increase of disintergin metalloprotease 10 (ADAM10) expression in oral squamous cell carcinoma. Cancer Lett 245: 33-43, 2007.

9. Kornberg LJ, Villaret D, Popp M, et al: Gene expression profiling in squamous cell carcinoma of the oral cavity shows abnormalities in several signaling pathways. Laryngoscope 115: 690-698, 2005.

10. Stokes A, Joutsa J, Ala-Aho R, et al: Expression profiles and clinical correlations of degradome components in the tumor microenvironment of head and neck squamous cell carcinoma. Clin Cancer Res 16: 2022-2035, 2010.

11. Takamune Y,Ikebe T, Nagano O, et al: ADAM-17 associated with CD44 cleavage and metastasis in oral squamous cell carcinoma. Virchows Arch 450: 169-177, 2007.

12. Xu Q, Liu X, Cai Y, Yu Y and Chen W: RNAi-mediated ADAM9 gene silencing inhibits metastasis of adenoid cystic carcinoma cells. Tumour Biol 31: 217-224, 2010. 
13. Yamano Y, Uzawa K, Shinozuka K, et al: Hyaluronan-mediated motility: a target in oral squamous cell carcinoma. Int J Oncol 32: 1001-1009, 2008.

14. Shiiba M, Nomura H, Shinozuka K, et al: Down-regulated expression of SERPIN genes located on chromosome 18q21 in oral squamous cell carcinomas. Oncol Rep 24: 241-249, 2010.

15. Lombardi DP, Geradts J, Foley JF, Chiao C, Lamb PW and Barrett JC: Loss of KAI1 expression in the progression of colorectal cancer. Cancer Res 59: 5724-5731, 1999.

16. Shinozuka K, Uzawa K, Fushimi K, et al: Downregulation of carcinoembryonic antigen-related cell adhesion molecule 1 in oral squamous cell carcinoma: correlation with tumor progression and poor prognosis. Oncology 76: 387-397, 2009.

17. Verona EV, Elkahloun AG, Yang J, Bandyopadhyay A, Yeh IT and Sun LZ: Transforming growth factor- $\beta$ signaling in prostate stromal cells supports prostate carcinoma growth by up-regulating stromal genes related to tissue remodeling. Cancer Res 67: 5737-5746, 2007.

18. Kveiborg M,Fröhlich C, Albrechtsen R, et al: A role for ADAM12 in breast tumor progression and stromal cell apoptosis. Cancer Res 65: 4754-4761, 2005.

19. Lendeckel U, Kohl J, Arndt M, Carl-McGrath S, Donat H and Röcken C: Increased expression of ADAM family members in human breast cancer and breast cancer cell lines. J Cancer Res Clin Oncol 131: 41-48, 2005.

20. Fröhlich C, Albrechtsen R, Dyrskjøt L, Rudkjær L, Ørntoft TF and Wewer UM: Molecular profiling of ADAM12 in human bladder cancer. Clin Cancer Res 12: 7359-7368, 2006.

21. Le Pabic H, Bonnier D, Wewer UM, et al: ADAM12 in human liver cancers: TGF- $\beta$-regulated expression in stellate cells is associated with matrix remodeling. Hepatology 37: 1056-1066, 2003.

22. Rocks N, Paulissen G, Quesada Calvo F, et al: Expression of a disintegrin and metalloprotease (ADAM and ADAMTS) enzymes in human non-small-cell lung carcinomas (NSCLC). Br J Cancer 94: 724-730, 2006

23. Carl-McGrath S,Lendeckel U, Ebert M, Roessner A and Rocken C: The disintegrin-metalloproteinases ADAM9, ADAM12, and ADAM15 are upregulated in gastric cancer. Int J Oncol 26: 17-24, 2005.

24. Kodama T, Ikeda E, Okada A, et al: ADAM12 is selectively overexpressed in human glioblastomas and is associated with glioblastoma cell proliferation and shedding of heparin-binding epidermal growth factor. Am J Pathol 165: 1743-1753, 2004.

25. Tian BL, Wen JM, Zhang M, Xie D, Xu RB and Luo CJ: The expression of ADAM12 (meltrin $\alpha)$ in human giant cell tumours of bone. Mol Pathol 55: 394-397, 2002.

26. Mori S, Tanaka M, Nanba D, et al: PACSIN3 binds ADAM12/ meltrin $\alpha$ and up-regulates ectodomain shedding of heparinbinding epidermal growth factor-like growth factor. J Biol Chem 278: 46029-46034, 2003.

27. Tanaka M, Nanba D, Mori S, et al: ADAM binding protein Eve-1 is required for ectodomain shedding of epidermal growth factor receptor ligands. J Biol Chem 279: 41950-41959, 2004.
28. Zahnow CA: ErbB receptors and their ligands in the breast. Expert Rev Mol Med 8: 1-21, 2006.

29. Ebi M, Kataoka H, Shimura T, et al: TGF $\beta$ induces proHB-EGF shedding and EGFR transactivation through ADAM activation in gastric cancer cells. Biochem Biophys Res Commun 402: 449-454, 2010.

30. Atfi A, Dumont E, Colland F, et al: The disintegrin and metalloproteinase ADAM1 2 contributes to TGF- $\beta$ signaling through interaction with the type II receptor. J Cell Biol 178: 201-208, 2007.

31. Bierie B and Moses HL: TGF- $\beta$ and cancer. Cytokine Growth Factor Rev 17: 29-40, 2006

32. Bierie B and Moses HL: Transforming growth factor $\beta$ (TGF- $\beta$ ) and inflammation in cancer. Cytokine Growth Factor Rev 21: 49-59, 2010.

33. Miettinen PJ, Ebner R, Lopez AR and Derynck R: TGF- $\beta$ induced transdifferentiation of mammary epithelial cells to mesenchymal cells: involvement of type I receptors. J Cell Biol 127: 2021-2036, 1994.

34. Qiao B, Johnson NW and Gao J: Epithelial-mesenchymal transition in oral squamous cell carcinoma triggered by transforming growth factor- $\beta 1$ is Snail family-dependent and correlates with matrix metalloproteinase-2 and -9 expressions. Int J Oncol 37: 663-668, 2010

35. Bartholin L, Destaing O, Forissier S, et al: FLRG, a new ADAM12associated protein, modulates osteoclast differentiation. Biol Cell 97: 577-588, 2005.

36. Thodeti CK, Albrechtsen R, Grauslund M, et al: ADAM12/ syndecan- 4 signaling promotes $\beta 1$ integrin-dependent cell spreading through protein kinase $\mathrm{C} \alpha$ and RhoA. J Biol Chem 278: 9576-9584, 2003.

37. Zhao Z, Gruszczynska-Biegala J, Cheuvront T, et al: Interaction of the disintegrin and cysteine-rich domains of ADAM12 with integrin $\alpha 7 \beta 1$. Exp Cell Res 298: 28-37, 2004.

38. Kang Q, Cao Y and Zolkiewska A: Metalloprotease-disintegrin ADAM12 binds to the SH3 domain of Src and activates Src tyrosine kinase in C2C12 cells. Biochem J 352: 883-892, 2000.

39. Kang Q, Cao Y and Zolkiewska A: Direct interaction between the cytoplasmic tail of ADAM12 and the Src homology 3 domain of p85a activates phosphatidylinositol 3-kinase in C2C12 cells. J Biol Chem 276: 24466-24472, 2001.

40. Fridman JS, Caulder E, Hansbury M, et al: Selective inhibition of ADAM metalloproteases as a novel approach for modulating ErbB pathways in cancer. Clin Cancer Res 13: 1892-1902, 2007.

41. Jacobsen J, Visse R, Sorensen HP, et al: Catalytic properties of ADAM12 and its domain deletion mutants. Biochemistry 47: 537-547, 2008 .

42. Kveiborg M, Jacobsen J, Lee MH, Nagase H, Wewer UM and Murphy G: Selective inhibition of ADAM12 catalytic activity through engineering of tissue inhibitor of metalloproteinase 2 (TIMP-2). Biochem J 430: 79-86, 2010. 